\title{
Effects of orienting tasks on sentence comprehension and cued recall
}

\author{
ROBERT E. TILL, DAVID R. CORMAK and PRESCOTT L. PRINCE \\ Davidson College, Davidson, North Carolina 28096
}

\begin{abstract}
In three experiments, cued recall of sentences was found to vary with the type of orienting task performed during sentence presentation. Retrieval cues referred to information probably inferred from the sentences. Each of the semantic tasks led to greater recall than did the nonsemantic task; this task effect occurred in a between-subjects design and in a within-subjects design. Furthermore, the use of a task-indicating signal after each sentence, in the within-subjects design, allowed the manipulation of the timing and type of orienting task. The task effect on recall appeared even with a delay of the task-indicating signal and/or the addition of an initial semantic task (performed prior to the indicated task). The findings suggest both a processing explanation and an interruption explanation of task effects.
\end{abstract}

During the comprehension process, listeners may make inferences based on what they hear. Memory for a sentence may interact with knowledge of the described event such that the resulting mental representation is something more elaborate than the original sentence. At retrieval, inferred information may serve as a cue for the reconstruction of the original encoding. This adaptation of the encoding specificity principle (Tulving \& Thomson, 1973) has provided the rationale for some recent studies of sentence memory (e.g., Barclay, Bransford, Franks, McCarrell, \& Nitsch, 1974; Till, Note 1). An important assumption in these studies has been that measures of the relative effectiveness of recall cues can be used to infer characteristics of the encoded representations of target items-characteristics based on information not explicitly identified during input.

For example, in the study by Till (Note 1), subjects were presented with sentences after being warned of a subsequent recall test. Their task was to comprehend each sentence and the event described in it. At the time of the test, a relevant (or irrelevant) cue was given as a prompt for recall of each sentence. For example, calendar was given as a cue for The secretary circled the dates. Relevant cues were generally found to be effective for recall, suggesting that, during sentence comprehension, subjects had inferred or encoded information that would later be contained in the cues. The study provided a powerful demonstration of intrasentence contextual effects (e.g., manuscript was a more effective cue than calendar for The proofreader circled the dates). And, it suggested that the relationship between the cues and sentences involved a wholistic comprehension of events as well as word associations.

Since the comprehension instructions were of a very

Requests for reprints should be sent to Robert E. Till, now at the Department of Psychology, Southern Methodist University, Dallas, Texas 75275 . general nature in the Barclay et al. (1974) and Till (Note 1) studies, little can be said about the critical activities essential to comprehension. How "automatic" are the inferences people make during comprehension? Do they occur when affective evaluations are made during comprehension? Such questions were not approached directly in the present study; however, it seemed worthwhile to manipulate the encoding processes during sentence presentation and to examine the subsequent cued recall. Through cue effectiveness, we could study the influence of encoding processes on inference and comprehension.

Encoding operations performed on an event are a major factor in memory performance. This point was emphasized by Jenkins (1974) and reiterated by Craik and Tulving (1975) in their account of the "new look" in memory research. Craik and Tulving suggested that a new "miniparadigm" has emerged, one in which "items are remembered not as presented stimuli acting on the organism, but as components of mental activity" (p. 292). For example, studies investigating the free recall of words have shown that the nature of an assigned orienting task has a major effect upon the subsequent amount of recall (Hyde \& Jenkins, 1969, 1973; Till \& Jenkins, 1973; Walsh \& Jenkins, 1973). Semantic tasks typically lead to good recall comparable to that which follows intentional learning. Furthermore, semantic tasks tend to reduce the amount of variance in recall; at least, the studies by Jenkins and his colleagues show a trend toward greater variance for intentional control groups than for semantic task groups. Thus, semantic orienting tasks permit greater control over the subject's activity, insure good recall, and produce greater homogeneity among recall scores. On the other hand, nonsemantic tasks which focus on formal properties of words lead to poor recall even when subjects know they will be tested. Similar task effects have been obtained in studies of sentence recall (e.g., MistlerLachman, 1974; Rosenberg \& Schiller, 1971). 
It is not our intention merely to extend the use of orienting tasks to a study of the cued recall of sentences. Rather, we are primarily interested in examining cued recall with its implications for the issue of "automaticity" of comprehension. With sufficient time, semantic tasks should facilitate comprehension and lead to good cued recall. Nonsemantic tasks should prevent or reduce comprehension and cause cued recall to be poor. These expectations are further supported by the fact that orienting tasks affect organization as well as recall. For example, Till, Diehl, and Jenkins (1975) presented subjects with a list of scrambled, highly associated word pairs. When subjects had performed a semantic task on each member of an associative pair, clustering in recall was high. But when subjects had performed a nonsemantic task on one or both members of the pair, clustering was considerably lower. That is, the performance of a nonsemantic task reduced the effectiveness of an intralist "cue" (the occurrence of one member of an associative pair in recall) for a related item from the list (the other member of the pair). Analogous results were expected in the present study: The performance of a nonsemantic task on a sentence would reduce the effectiveness of an appropriate extralist cue for recall of the sentence.

Subjects in Experiments I and II performed one of three orienting tasks on each sentence they heard. Two of these tasks were semantic: a pleasantness-rating task and a comprehension-response task (requiring a short response to the sentence meaning). The third task was nonsemantic and required an estimation of the number of words contained in a sentence: a wordestimation task. We reasoned that the comprehensionresponse task was most like the normal comprehension process; cued recall following this task was expected to be good. The pleasantness-rating task seemed less similar to normal comprehension because of its emphasis on a forced evaluation. Nevertheless, it makes subjects focus on sentence meaning and probably permits inferences to be made during the evaluation. Thus, cued recall following the pleasantness-rating task was expected to be just as good as after the comprehensionresponse task. In the case of the word-estimation task, it was expected that subjects would be less likely to deal with meaning or to make inferences; cued recall should be poor.

In their concluding remarks, Craik and Tulving (1975) recommended the study of memory for items used in various combinations of orienting tasks. A few studies of this sort have already demonstrated the effects of tasks performed singly and in combination (e.g., Walsh \& Jenkins, 1973); the approach provides a valuable way to control, delay, or interrupt the encoding process. Accordingly, subjects in Experiment III performed orienting tasks only after a brief delay and/or performed an additional initial task on each sentence. By imposing such task and timing constraints, it was possible to study the limits on the task effects observed in Experiments I and II.

\section{EXPERIMENT I}

In the first experiment, each subject performed only one of the three orienting tasks. Thus, the experiment involved a between-groups design in which one group performed the comprehension-response task, a second group performed the pleasantness-rating task, and a third group performed the nonsemantic wordestimation task.

\section{Method}

Subjects. All subjects were undergraduate students enrolled in an introductory psychology class at Davidson College. Each received course credit for participation. Subjects were tested in small groups, and within each group they were randomly assigned to one of the task conditions. A total of 45 subjects was tested, 15 subjects per task condition.

Materials. Twenty-one sentences were constructed such that certain inferences or elaborations of meaning would be quite probable for the undergraduate students. The following examples were used in the experiment: (1) The children watched the program. (2) The hostess raised her glass in the air. (3) The pupil carefully positioned a thumbtack on the chair. Sentences ranged in length from 5 to 10 words; approximately half of the list consisted of five-word sentences. The structure of all the sentences can be easily summarized in terms of cases: human agent + verb + object and/or one other case (such as location or manner). One randomized presentation order was used for all subjects.

A list of 21 recall cues, one for each test sentence, was also prepared. Each cue was a noun referring to information that might be inferred from the sentence, such as an object or instrument involved in the described event or the purpose or social significance of the event. For example, the following cues correspond to the test sentences given above: (1) television, (2) toast, and (3) prank. As in the Till (Note 1) study, a cue word was chosen primarily to represent information inferred from the total event described in a sentence rather than to trigger a verbal association with one of the words in the sentence. Three orders of cues were prepared. These were random arrangements, except for the constraint that each cue appear in a different one-third of the list. Thus, television appeared near the beginning of one cue list, near the middle of another, and near the end of the remaining order. None of the cue orders was related to the presentation order of the sentences.

Apparatus. Test materials and general instructions were presented with a Panasonic RS-740-US tape recorder and a Panasonic RS-281-S amplifier with attached speakers. Instructions specific to each orienting task were printed at the top of subjects' answer sheets. Different answer sheets were prepared for each orienting task condition.

Procedure. Each group of subjects was brought into the laboratory and given a general orientation to the experiment. Answer sheets were randomly distributed to subjects from a single stack containing the three types of answer sheet. Thus, the assignment of task conditions to subjects was random within each test group. The tape recorder was started and subjects heard instructions to take approximately $2 \mathrm{~min}$ to read the task description at the top of the answer sheet. These descriptions were repetitious and provided examples, but they can be summarized as follows:

In the comprehension-response task, subjects were to think about the event described in each sentence and the things that 
might be involved in this event. They then wrote down a word as a response to their interpretation of the sentence.

In the pleasantness-rating task, subjects were instructed to think about the meaning of each sentence and to decide whether the sentence described something that would be "pleasant" or "unpleasant" in their opinion. Based on their rating, subjects placed a checkmark in one of the two columns on their answer sheet.

In the wordestimation task, subjects were asked to estimate the number of words in each sentence. They were instructed to estimate, rather than count, the words, so that they could check one of three categories, " 5 ," " 7 ," or "9," whichever seemed closest to their estimate.

Subjects were told that they would be questioned about the sentences later and that further instructions would be given at that time. The sentences were presented at the rate of one new sentence every $15 \mathrm{sec}$. Each sentence was presented and immediately repeated to minimize errors of speech perception. Each subject then performed his assigned task.

After presentation of the sentences, subjects were given answer booklets and asked to number the pages. At the end of this 2 -min period, instructions about the recall test were given. One of the three cue orders was randomly selected, and the cues were presented at the rate of one every $30 \mathrm{sec}$. Again, for clarity, each cue was presented and immediately repeated. Subjects were told that each cue would be related to a sentence presented earlier and that some of the cues would be more effective than others. Responses were to be written on the appropriate page in the answer booklet. Guesses, omissions, and repetitions were considered permissible. Subjects were also told to use the exact wording when possible.

Scoring. Subjects' responses were scored on two levels of acceptability. Level 1 acceptability characterized sentences recalled verbatim, as well as sentences which contained reasonably synonymous substitutions or omissions of partially redundant information. Level 2 acceptability was more inclusive, incorporating all the transformations listed above plus a few others. For example, sentences with omissions of nonredundant information were accepted as long as the agent, verb, and some of the additional information were specified. Typically, these were sentences with an adverb or some other significant word missing. Responses not acceptable at either level were relegated to the "other" category. One other classification, intralist intrusion, was defined as an acceptable sentence written in response to a cue designated as a prompt for some other sentence from the list.

The criteria for acceptability were studied and discussed by two judges. Their independent ratings of responses were then made without awareness of the task condition from which a particular response came. Interrater reliabiltiy coefficients for the two levels of the three task conditions ranged from .96 to 99. Scoring discrepancies were resolved to provide a single set of scores for analysis.

\section{Results and Discussion}

Three groups were formed by pooling the data of subjects who performed the same orienting task (irrespective of the cue order during recall). The amount of recall in these three task conditions was compared; means and standard deviations are presented in Table 1.

A single-factor analysis of variance was performed on the Level 1 recall data of the three task conditions. The overall effect due to orienting tasks was clearly significant $[F(2,42)=25.46, p<.001]$. Individual comparisons were made between the task conditions. Cued recall in the word-estimation group was found to be significantly lower than recall in the comprehensionresponse group $[\mathrm{F}(1,42)=37.80, \mathrm{p}<.001]$ and lower
Table 1

Cued Recall of Sentences in Experiment I

\begin{tabular}{lccc}
\hline & \multicolumn{3}{c}{ Task Condition } \\
\cline { 2 - 4 } \multicolumn{1}{c}{ Variable } & $\begin{array}{c}\text { Compre- } \\
\text { hension } \\
\text { Response } \\
\text { (Group 1) }\end{array}$ & $\begin{array}{c}\text { Pleasant- } \\
\text { ness } \\
\text { Rating } \\
\text { (Group 2) }\end{array}$ & $\begin{array}{c}\text { Word } \\
\text { Estima- } \\
\text { tion } \\
\text { (Group 3) }\end{array}$ \\
\hline Mean Recall (Level 1) & 8.60 & 8.67 & 2.20 \\
SD & 2.77 & 3.04 & 2.73 \\
Mean Recall (Level 2) & 10.60 & 10.27 & 2.67 \\
SD & 2.47 & 3.43 & 3.37 \\
Intralist Intrusions & .67 & .73 & .40 \\
SD & .82 & .80 & .91 \\
Other & .40 & .27 & .40 \\
SD & .83 & .46 & .63 \\
\hline
\end{tabular}

Note-Fifteen subjects participated in each group.

than recall in the pleasantness-rating group $[F(1,42)=$ $38.59, \mathrm{p}<.001]$. Recall following the comprehensionresponse task and the pleasantness-rating task did not differ $(p>.10)$.

An analysis performed on the Level 2 recall data yielded similar results: an overall effect due to task $[F(2,42)=33.16, p<.001]$; lower recall in the wordestimation group than in either the comprehensionresponse group $[F(1,42)=51.83, p<.001]$ or the pleasantness-rating group $[\mathrm{F}(1,42)=47.56, \mathrm{p}<.001]$; and no difference between the comprehension-response and pleasantness-rating groups $(p>.10)$.

An analysis of variance performed on the intralist intrusions showed no effect due to orienting task $(p>.10)$. Similarly, the analysis on nonacceptable ("other") responses indicated no effect due to task $(p>.10)$.

It was also informative to examine item analyses of the materials. That is, the percentage of subjects recalling a particular sentence on cue was examined as a function of the task condition. To use one of the strongest examples, The children watched the program was recalled in response to television by $87 \%$ of the subjects in the comprehension-response group, by $80 \%$ of the pleasantness-rating group, and by only $20 \%$ of the word-estimation group (based on Level 2 scoring). Furthermore, the percentage of subjects in the wordestimation group recalling a particular sentence on cue was lower than the percentage in the comprehensionresponse group and lower than the percentage in the pleasantness-rating group for 20 of the 21 sentences (based on Level 2 scoring).

These findings support the hypothesis that orienting tasks influence comprehension and recall. Semantic tasks which emphasized or permitted inferential activity during comprehension led to greater cued recall than did the nonsemantic task. During sentence presentation, subjects in the nonsemantic group were much less likely to relate information in a sentence to their knowledge of the described event. Thus, a cue which focused on that relationship was of little use later for recall of the sentence. 


\section{EXPERIMENT II}

The second experiment was an attempt to replicate the differential effects of the orienting tasks within subjects by means of a task-cuing procedure (cf. Till \& Jenkins, 1973). The subject was required to change tasks unpredictably from sentence to sentence. With this procedure, it was possible to eliminate concern for the effect of overall "set" and to insure greater similarity in all subjects' initial orientation to a sentence (since the task-indicating cue was given only after the sentence had been said twice). Furthermore, the repeatedmeasures design provided a sensitive test of the generality of the task effects for individuals. As in Experiment I, it was predicted that subjects would recall more comprehension-response and pleasantness-rating sentences than word-estimation sentences. Of course, in this experiment, the pattern of results was expected within subjects.

\section{Method}

Subjects. The 29 subjects of Group 4 were drawn from the same population as used in Experiment $I$ and were given similar credit for participation.

Materials and apparatus. The sentence list and the three cue orders of Experiment I were also used in this experiment. All instructions and test materials were presented to individual subjects with a Panasonic RS-740-US tape recorder and Koss Pro-4AA headphones.

Procedure. Subjects were tested individually. After a brief orientation, the subject was seated and given the headphones and an answer sheet. The tape recorder was started and the subject listened to instructions explaining the three orienting tasks. Task descriptions, scoring procedure, recall instructions, and other procedural details were the same as in the first experiment except for instructions about the task-indicating cues.

In this experiment, each sentence was presented, repeated, and followed by a cue letter, " $A$," "B," or " $C$," indicating which task was to be performed on the preceding sentence. The letter came approximately $.5 \mathrm{sec}$ after the repeated sentence, leaving about $10 \mathrm{sec}$ for execution of the indicated task. Each subject performed a given task on 7 of the 21 sentences. Answer sheets were divided into three columns, one for each task. At the top of each column, a cue letter was identified with a particular task (e.g., "A" might refer to pleasantness-rating). For the group of subjects as a whole, cue letters were assigned to tasks in six different ways; this served to counterbalance any effect due to the interaction of a task with specific sentences.

\section{Results and Discussion}

The data from all subjects in the experiment were pooled. That is, there was no analysis on the basis of the six letter-by-task assignments or the three orders of recall cues. Interrater reliability coefficients for the two levels of total recall were .97 and .99 . As in Experiment $I$, discrepancies were resolved before the data analysis began. Table 2 presents the mean values of the total score and the three scores indicating the amount of cued recall following each task.

An analysis of variance performed on the Level 1 recall data indicated a significant overall effect due to tasks $[\mathrm{F}(2,56)=30.11, \mathrm{p}<.001]$; the mean square
Table 2

Cued Recall of Sentences in Experiment II

\begin{tabular}{|c|c|c|c|c|}
\hline \multirow[b]{2}{*}{ Variable } & \multicolumn{4}{|c|}{ Cued-Task Condition } \\
\hline & $\begin{array}{l}\text { Compre- } \\
\text { hension } \\
\text { Response }\end{array}$ & $\begin{array}{l}\text { Pleasant- } \\
\text { ness } \\
\text { Rating }\end{array}$ & $\begin{array}{l}\text { Word } \\
\text { Estima- } \\
\text { tion }\end{array}$ & $\begin{array}{c}\text { All } \\
\text { Tasks } \\
\text { (Total) }\end{array}$ \\
\hline Mean Recall (Level 1) & 3.69 & 3.59 & 1.55 & 8.83 \\
\hline Mean Recall (Level 2) & 4.17 & 3.86 & 1.66 & 9.69 \\
\hline Possible & 7 & 7 & 7 & 21 \\
\hline
\end{tabular}

Note-Group 4 consisted of 29 subjects.

error (MSe) was 1.40. Individual comparisons using this overall MSe were made between the task conditions. Subjects recalled significantly fewer word-stimation than comprehension-response sentences $[F(1,56)=$ 47.34, $\mathrm{p}<.001]$ and fewer wordestimation than pleasantness-rating sentences $[\mathrm{F}(1,56)=42.87$, $\mathrm{p}<.001]$. Recall of comprehension-response and pleasantness-rating sentences did not differ $(p>.10)$.

An analysis performed on the Level 2 data yielded a similar overall task effect $[F(2,56)=42.02, p<.001$, $\mathrm{MSe}=1.30]$. Individual comparisons using this overall MSe showed lower recall of word-estimation sentences than either comprehension-response $[\mathrm{F}(1,56)=70.68$, $\mathrm{p}<.001]$, or pleasantness-rating sentences $[F(1,56)=$ $54.32, \mathrm{p}<.001]$, and no difference in the recall of comprehension-response and pleasantness-rating sentences $(p>.10)$.

Analyses performed on the intralist intrusions and the nonacceptable responses showed no differential effect due to tasks (in both cases, $p>.10$ ).

These findings support the hypothesis of task effects and provide a within-subjects replication of the first experiment. Comprehension and cued recall were strongly influenced by the orienting task subjects performed. Furthermore, the magnitude of the task effect was seen in the recall of the individual. Consider the number of subjects who recalled fewer wordestimation than comprehension-response sentences as well as fewer word-estimation than pleasantness-rating sentences; this pattern in recall was seen for 26 of the 29 subjects (based on Level 2 scoring).

\section{EXPERIMENT III}

The first two experiments clearly showed that the nonsemantic task led to poor recall. Even for subjects in Group 4 who processed two-thirds of the sentences semantically, sentences used in the nonsemantic task were poorly recalled. Such results suggest that meaningful processing is not automatic or that the task in some way interferes with comprehension. In order to understand this task effect better, we decided to assign combinations of tasks and to manipulate the timing of the tasks. In effect, we wanted to determine conditions that would reduce or eliminate the task effect.

If subjects in Group 4 typically delayed their sen- 
tence processing until they heard the task-indicating cue, then an additional initial semantic task might make them start the sentence processing earlier. Thus, some semantic processing would have occurred before the subject began the cued task. With this arrangement, some of the sentences would be used in a semanticnonsemantic combination of tasks. In accord with the processing view of Walsh and Jenkins (1973), one would expect recall in this case to be similar to that which follows the performance of a semantic task alone. Furthermore, the processing view would lead one to predict that the arrangement described here would considerably reduce the task effect on recall seen in Experiment II. The occurrence of some semantic processing would be the equalizing factor.

Alternatively, it may be that subjects in Group 4 did begin meaningful processing of the sentences before task-indicating cues were given. If so, an extra semantic task would simply specify a particular type of initial meaningful processing, but would not necessarily be an improvement over the subject's own activity. Furthermore, the task effect on recall would probably be similar with or without the extra task. This could mean that, for both conditions, the nonsemantic task interferes with the earlier semantic processing, perhaps preventing consolidation of activated information; or it could be that neither condition provides sufficient time to execute a meaningful processing of the material.

Two other conditions were included in this experiment. They differed from the condition described above in that the task-indicating cue was presented only after a short delay. With this delay between the sentence presentation and the cue letter, subjects should have sufficient time for meaningful processing-whether spontaneous or specified. One group performed the extra semantic task while waiting for the cue to indicate the main orienting task; the other group performed no specific task prior to the cue. It was important to determine whether the task effect on recall would be similar for these two groups despite the fact that one group performed the extra task. Furthermore, since the timing allowed for a more comfortable completion of an initial task and a cued task, it was possible to expect support for the consolidation-interruption or processing view of recall. If the familiar task effect on recall appeared, there would be support for the consolidationinterruption notion. If the task effect were reduced, there would be support for the processing view.

\section{Method}

Subjects. Subjects were drawn from the same population as used in Experiment I and given similar credit for participation. They were tested in small groups; each group was randomly assigned to one of the three experimental conditions. Group 5 consisted of 20 subjects. Group 6 had 20 subjects, and Group 7 included 19 subjects.

Materials and apparatus. The sentence list and the three cue orders of Experiment I were also used in this experiment. All instructions and test materials were presented to subjects by means of a tape recorder. The original and a modified version of the sentence list were used in this experiment. The modified list was recorded with the cue letters delayed such that subjects would have only the last $5 \mathrm{sec}$ of the $15-\mathrm{sec}$ interval in which to perform the cued task. Answer sheets were similar to those of Experiment II, except for an added section in which subjects could mark their ratings during an initial (extra) task.

Procedure. Procedural details were similar to those of Experiment II, except that subjects were tested in small groups. Task descriptions, scoring procedure, and instructions about taskindicating cues and recall were the same as in Experiment II except for the changes noted below.

Subjects in Group 5 were asked to perform an initial interestrating task on each sentence during the brief interval in which the sentence was being repeated and before the main task was indicated by the cue letter. Subjects were instructed to think about the meaning of each sentence and to decide whether it seemed "interesting" or "uninteresting" in their opinion. They then placed a checkmark in one of two columns on their answer sheet. Subjects were warned that this task was important, although very little time would be allotted for it. Instructions emphasized quick judgments to be made before the cued task was begun.

Subjects in Group 6 also performed the initial interest-rating task. However, they heard the modified sentence list in which the cue letters were delayed, allowing nearly equal time for performance of the initial task and the cued task.

Subjects in Group 7 heard the modified sentence list in which the cue letters were delayed. They performed no initial task, but simply waited for the cue letter to indicate the appropriate task.

\section{Results and Discussion}

As in the other experiments, there was no analysis on the basis of letter-by-task assignment or order of recall cues. Scores indicating the amount of cued recall following each of the three cued tasks were obtained for every subject. For each group of subjects, singlefactor analyses of variance were performed on the Level 1 recall following the cued tasks as well as on the Level 2 recall. Since the results based on Level 1 and Level 2 recall data did not differ, only Level 2 data are reported in this experiment. Interrater reliability coefficients for the nine Level 2 ratings ( 3 task conditions by 3 experimental conditions) ranged from .95 to .99 . Table 3 presents the mean scores for the three experimental conditions: extra-task condition (Group 5), delay + extra-task condition (Group 6), and delay condition (Group 7).

For Group 5, the analysis of variance indicated that the overall effect due to tasks was highly significant

Table 3

Cued Recall of Sentences in Experiment III

\begin{tabular}{|c|c|c|c|}
\hline \multirow[b]{2}{*}{ Mean Recall } & \multicolumn{3}{|c|}{ Cued-Task Condition } \\
\hline & $\begin{array}{l}\text { Compre- } \\
\text { hension } \\
\text { Response }\end{array}$ & $\begin{array}{l}\text { Pleasant- } \\
\text { ness } \\
\text { Rating }\end{array}$ & $\begin{array}{l}\text { Word } \\
\text { Estima- } \\
\text { tion }\end{array}$ \\
\hline $\begin{array}{l}\text { Extra-Task Condition } \\
\text { (Group } 5, N=20 \text { ) }\end{array}$ & 4.65 & 3.95 & 2.40 \\
\hline $\begin{array}{l}\text { Delay + Extra-Task Condition } \\
(\text { Group 6, N }=20)\end{array}$ & 3.95 & 3.15 & 2.65 \\
\hline $\begin{array}{l}\text { Delay Condition } \\
\text { (Group } 7, N=19 \text { ) }\end{array}$ & 3.84 & 2.89 & 1.84 \\
\hline
\end{tabular}

Note-Based on Level 2 recall data only. 
$[F(2,38)=17.33, \mathrm{p}<.001, \mathrm{MSe}=1.53]$. Although all sentences received a quick initial rating for interest, the subsequent cued task exerted a strong influence on which sentences were recalled. Individual comparisons using the overall MSe were made between the task conditions, and the outcome resembled that of Experiment II. Subjects recalled significantly fewer wordestimation than comprehension-response sentences $[F(1,38)=33.08, p<.001]$ and fewer word-estimation than pleasantness-rating sentences $[F(1,38)=15.70$, $\mathrm{p}<.001]$. Recall of comprehension-response and pleasantness-rating sentences did not $\operatorname{differ}[F(1,38)=$ $3.20, p>.05]$.

For Group 6 , the analysis of variance showed that the overall effect due to tasks was significant $[\mathrm{F}(2,38)=$ $4.53, \mathrm{p}<.025, \mathrm{MSe}=1.90]$. Since the performance of the cued task was considerably delayed, subjects rated sentences for interest at a comfortable pace (compared to Group 5). Yet the cued task still influenced the subsequent cued recall. Individual comparisons using the overall MSe were made between the task conditions. Subjects recalled significantly fewer word-estimation than comprehension-response sentences $[F(1,38)=8.89$, $\mathrm{p}<.01]$. However, recall of pleasantness-rating sentences was at an intermediate level; it was not significantly different from word-estimation recall $(p>.10)$, nor was it different from comprehension-response recall $[F(1,38)=3.37, \mathrm{p}>.05]$.

For Group 7, the analysis of variance revealed that the overall task effect was highly significant $[F(2,36)=$ $19.41, \mathrm{p}<.001, \mathrm{MSe}=.98]$. Individual comparisons using the overall MSe indicated that subjects recalled more comprehension-response than pleasantness-rating sentences $[F(1,36)=8.70, p<.01]$ and more pleasantness-rating than word-estimation sentences $[F(1,36)=10.74, p<.005]$. And, of course, they recalled more comprehension-response than wordestimation sentences $[F(1,36)=38.77, p<.001]$. Recall of pleasantness-rating sentences was again at an intermediate level, but this time it differed significantly from the other two task conditions.

These results appear to support both a consolidationinterruption explanation and the processing explanation of task effects. None of the conditions of this experiment led to an elimination of the task effect on recall, not even those allowing sufficient time for initial meaningful processing of the sentences. A cued task that is nonsemantic apparently interrupts the ongoing meaningful processing and hinders later recall. However, there was evidence that the task effect was reduced by requiring subjects to perform an initial semantic task. For example, in Group 6 recall of word-estimation sentences was not significantly different from recall of pleasantness-rating sentences. Furthermore, in Groups 5 and 6 , the extra task appears to have improved the recall of word-estimation sentences; mean recall was higher than in Group 4 or 7. The mere performance of a semantic task during some portion of the processing interval is not sufficient to eliminate the task effect; hence, a simple prediction based on the Walsh and Jenkins (1973) processing view is not accurate. However, it appears that combining a semantic task with the nonsemantic word-estimation task may reduce the magnitude of the task effect. Thus, the processing and interruption hypotheses are both partially supported by the data of this experiment.

\section{FURTHER ANALYSIS AND DISCUSSION}

The results of Experiment III indicated that the extra semantic task improved word-estimation recall, although it had little effect on the other two recall scores. Furthermore, there was some evidence to suggest that the delay variable hindered semantic task recall, while it facilitated nonsemantic task recall. In order to examine these two variables in combination, we decided to recast the results of Experiments II and III as separate 2 by 2 matrices-one for each cued-task condition. (The authors recognize the inelegance of comparing the individually tested subjects of Group 4 with Groups 5,6 , and 7 , who were tested in small groups; however, pilot research suggested that the testing variable was not a significant factor.) Table 4 presents the mean cued recall for each cued-task condition as a function of the delay and extra-task factors.

The data of Table 4 were analyzed in separate twoway analyses of variance. Thus, for each cued-task condition, we examined the effect of delay and the effect of the extra semantic task. For the comprehensionresponse task, no significant effects emerged. For the pleasantness-rating task, the delay variable caused a significant difference in recall $[F(1,84)=7.21, p<.01]$; the extra-task variable and the interaction were not significant. For the word-estimation task, the extra-

Table 4

Cued Recall as a Function of Cued-Task, Delay, and Extra-Task Variables

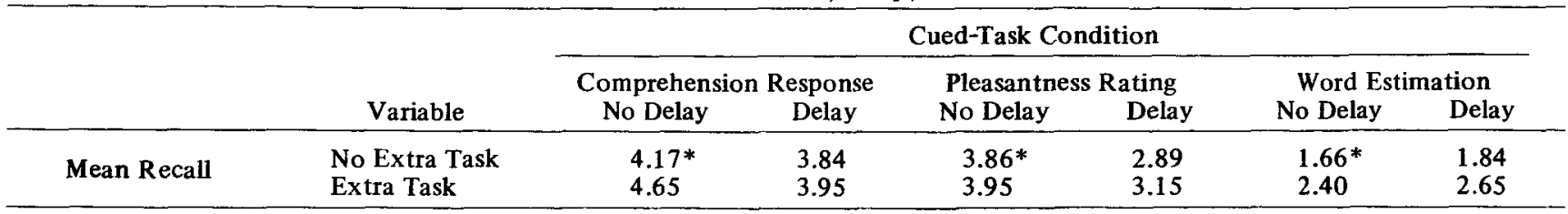

Note-Based on Level 2 recall data only.

${ }^{*}$ Data are from Group 4 of Experiment II; all other data are taken from Experiment III. 
task variable produced a significant recall difference $[F(1,84)=5.79, p<.025]$; no other effects were significant.

These analyses confirm the existence of certain trends in the data of Experiment III. For example, it is clear that the initial semantic task improved cued recall of sentences used in the word-estimation task. This initial task had no effect on sentence recall for the other two cued-task conditions. Apparently, it makes no difference whether a subject performs one or two semantic tasks, even though two tasks in combination may involve more processing time. (At least, this is true where the additional task is interest rating.) On the other hand, whether or not a subject performs at least one semantic task is critical. Recall following the cued word-estimation task improved when an initial semantic task was assigned. This suggests that in the wordestimation condition, subjects tend not to focus on meaning while waiting for the task-indicating cue. Thus, the assigned processing before the cued task contributes to greater recall than spontaneous processing before the cued task.

Initially, it was expected that delay of the cued task would serve to lower recall in the semantic task conditions. If the comprehension-response task or the pleasantness-rating task required several seconds of processing time, then delay of the task might leave too little time for a proper performance of the task. The results of the additional analyses suggest that the two semantic tasks differ in this respect: the comprehensionresponse task is performed quickly, while the pleasantness-rating task suffers from the shorter interval in the delay condition. An account of this sort seems plausible given the nature of the two semantic tasks.

In the case of the word-estimation task, a reversal was expected. We reasoned that the delay of this task would leave more time for meaningful processing, whether assigned or spontaneous. This expectation proved to be wrong. While mean recall did reflect a reversal, the effect was not a significant one. Consider an example of word-estimation recall from Table 4: Subjects in the delay condition with no extra task (Group 7) waited several seconds before they performed the word-estimation task (when it was indicated). Their mean recall of 1.84 sentences is less than would be expected of subjects who engaged in spontaneous semantic processing during the delay interval. That is, the level of recall does not support the hypothesized meaningful processing during the delay interval. In fact, there is evidence that the subjects were involved in a rather different activity. When they were questioned after the experiment, each subject in Group 7 reported some strategy for guessing the task that would be cued at the end of the delay interval. It is important to note that few reported that they began to perform one of the tasks; most were concerned with trying to determine which task would be cued. Thus, comprehension was not an automatic response to the presentation of a sentence.
In conclusion, some general points should be emphasized. In all three experiments, the amount of cued recall was influenced by the orienting tasks subjects performed on the sentences. Either semantic task led to greater recall than did the nonsemantic task; this task effect occurred even when the cued tasks were combined with an additional semantic task. Although the comprehension-response task and the pleasantnessrating task differed in terms of the specific meaningful responses required of subjects, and in terms of processing time, they were similar in that both enabled subjects to draw inferences from the sentences. As in the Till (Note 1) study, cues based on probable inferences were generally effective for sentence recall, provided the sentences had been processed semantically. It seems likely that other tasks requiring the subject to focus on the meaning of a sentence would also permit inferences to be made, and would lead to good cued recall.

The fact that appropriate retrieval cues were of little use in the nonsemantic conditions of the present study suggests that subjects had very little information in memory about the sentences they processed. Furthermore, associations between the sentences and retrieval cues were not automatic. That is, the effectiveness of a cue for a sentence does not depend solely on whether the subject knows the relationship between the sentence and cue, nor does it depend only on whether the cue is a reasonable inference from the sentence. Rather, the value of the cue seems to result from the subject's activation of knowledge about the cue-sentence relationship during sentence comprehension. A subject comprehends, and perhaps evaluates, the event underlying a sentence and later finds that the cue refers to something he knows to be related to this event. Thus, the subject's "experience" of the sentence is critical; his activity with regard to the sentence influences the effectiveness of a potential retrieval cue.

Orienting tasks provide an important means of manipulating encoding processes. The approach taken in the present study is compatible with Craik and Tulving's (1975) "miniparadigm," and allows for further research on memory through methods of control, combination, delay, or interruption of encoding processes.

\section{REFERENCE NOTES}

1. Till, R. E. Sentence memory prompted with inferential recall cues. Submitted for publication, 1976.

\section{REFERENCES}

Barclay, J., Bransford, J., Franks, J., McCarrell, N., \& NITsCH, K. Comprehension and semantic flexibility. Journal of Verbal Learning and Verbal Behavior, 1974, 13, 471-481.

Craik, F., \& Tulving, E. Depth of processing and the retention of words in episodic memory. Journal of Experimental Psychology: General, 1975, 104, 268-294.

Hyde, T., \& JENKINS, J. Differential effects of incidental tasks on the organization of recall of a list of highly associated words. Journal of Experimental Psychology, 1969, 82. 472-481. 
Hyde, T., \& Jenkins, J. Recall for words as a function of semantic, graphic, and syntactic orienting tasks. Joumal of Verbal Learning and Verbal Behavior, 1973, 12, 471-480.

JENkins, J. Remember that old theory of memory? Well, forget it! American Psychologist, 1974, 29, 785-795.

Mistler-Lachman, J. Depth of comprehension and sentence memory. Journal of Verbal Learning and Verbal Behavior, $1974,13,98-106$.

Rosenberg, S., \& Schiller, W. Semantic coding and incidental sentence recall. Journal of Experimental Psychology, 1971, 90, 345-346.

Till, R., DiehL, R., \& Jenkins, J. Effects of semantic and nonsemantic cued orienting tasks on associative clustering in free recall. Memory \& Cognition, 1975, 3, 19-23.
Till, R., \& Jenxins, J. The effects of cued orienting tasks on the free recall of words. Journal of Verbal Learning and Verbal Behavior, 1973, 12, 489.498.

Tulving, E., \& Thomson, D. Encoding specificity and retrieval processes in episodic memory. Psychological Review, 1973, 80, 352-373.

WALsh, D., \& Jenkins, J. Effects of orienting tasks on free recall in incidental learning: "Difficulty," "effort," and "process" explanations, Journal of Verbal Learning and Verbal Behavior, 1973, 12, 481-488.

(Received for publication March 1, 1976; revision received April 20, 1976.) 